Acta Botanica Mexicana (2003), 62: 1-8

\title{
TRES NUEVAS LOCALIDADES DE TIGRIDIAS ENDÉMICAS DE MÉXICO: TIGRIDIA BICOLOR, T. MATUDAE Y T. VANHOUTTEI SSP. ROLDANII
}

\author{
Aarón Rodríguez \\ LUIS Ortiz-Catedral \\ Departamento de Botánica y Zoología \\ Universidad de Guadalajara \\ Apartado postal 139 \\ 45110 Zapopan, Jalisco \\ Y \\ Edmund Heaton \\ 46 Manor Drive \\ Kingskerswell \\ Devon, TQ12 SHD \\ England
}

\section{RESUMEN}

México es el centro de diversificación del género Tigridia (Tigridieae: Iridaceae). El accidentado relieve y la diversidad climática presentes en el país han favorecido el desarrollo de endemismos en este grupo. En los veranos de 1996 y 2000 se llevaron a cabo colectas botánicas a fin de ampliar el conocimiento de la distribución geográfica de la tribu Tigridieae y obtener semillas, bulbos y hojas para el estudio filogenético del grupo. Como resultado de este trabajo, se localizaron nuevas poblaciones de T. bicolor Molseed, T. matudae Molseed y T. vanhouttei ssp. roldanii Molseed en Oaxaca, Morelos y Puebla respectivamente. Estos taxones solo se conocían de la localidad tipo. Las nuevas localidades se presentan junto con observaciones de campo y aspectos de fenología de las especies.

Palabras clave: Iridaceae, México, Tigridia.

\begin{abstract}
Mexico is the diversity center of the genus Tigridia (Tigridieae: Iridaceae). Its irregular topography and climatic diversity have generated a high number of endemics in this group of plants. We conducted collecting expeditions in Mexico during the summers of 1996 and 2000. The goals of the expeditions were to widen the known geographical distribution of Tigridieae and to collect leaf material, bulbs and seeds for phylogenetic studies of this group of plants. As a result, we found new populations of $T$. bicolor Molseed, T. matudae Molseed and T. vanhouttei ssp. roldanii Molseed, in Oaxaca, Morelos, and Puebla respectively. These taxa were known only from their type localities. These new localities are presented together with field observations and aspects of phenology.
\end{abstract}

Key words: Iridaceae, Mexico, Tigridia. 


\section{INTRODUCCIÓN}

Tigridia Jussieu es uno de los géneros de Iridaceae mejor representados en México. En el país se encuentran alrededor de 35 especies, de las cuales 25 son endémicas, cinco crecen en Guatemala y cinco más en Ecuador y Perú (Henrich y Goldblatt, 1994; Goldblatt et al., 1998). Su discontinua distribución geográfica en América del Norte y del Sur ofrece un modelo ideal para explorar aspectos biogeográficos. Las especies de Tigridia se distinguen por presentar seis tépalos diferenciados en dos series de tres, filamentos connados y tres ramas del estilo bífidas y opuestas a los estambres.

El género Tigridia constituye un ejemplo de radiación adaptativa. Los bajos niveles de variación en las secuencias de nucleótidos de miembros mexicanos y guatemaltecos sugieren que la radiación adaptativa en Tigridia y géneros afines es reciente y fue influenciada por la diversidad topográfica y climática, así como por la interacción plantapolinizador (Rodríguez, 1999). La variación en color, tamaño y forma del perianto en las especies de Tigridia indican que la adaptación a los polinizadores ha sido un factor importante en su diversificación. Se ha sugerido polinización por colibríes para T. flammea (Lindl.) Ravenna, T. immaculata (Herb.) Ravenna, T. innusitata (Cruden) Ravenna y $T$. ortantha (Lem.) Ravenna (Cruden, 1971); por moscas para T. molseediana Ravenna, $T$. vanhouttei (Baker) Espejo \& López-Ferrari ssp. vanhouttei, T. alpestris Molseed ssp. alpestris (Molseed, 1970), T. ehrenbergii (Schltdl.) Molseed ssp. ehrenbergii y T. bicolor Molseed (Cruden, 1975); por mariposas para T. pavonia (L.f.) DC. y por abejas para el complejo de T. mexicana Molseed (Molseed, 1970). A pesar de que los síndromes de polinización son claramente distinguibles, no existen estudios relativos a la determinación de los polinizadores involucrados.

Otro factor importante en la diversificación de Tigridia es el accidentado relieve de México. La topografía y la variedad climática han favorecido el desarrollo de endemismos. Muchas especies se conocen sólo de su localidad tipo. En esta situación se encuentran T. catarinensis Cruden, T. chrysantha Cruden \& S. J. Walker, T. ehrenbergii (Schltdl.) Molseed ssp. flaviglandifera Cruden, T. estelae López-Ferrari \& Espejo, T. hallbergii Molseed ssp. Iloydii Cruden, T. hintonii Molseed, T. mortonii Molseed, T. purpusii Molseed, T. tepoxtlana Ravenna y T. venusta Cruden (Molseed, 1970; Cruden, 1975; López-Ferrari y Espejo-Serna, 1994; Espejo-Serna y López-Ferrari, 1996a, 1996b; Rodríguez et al., 1996). Tigridia illecebrosa Cruden se conocía sólo de la zona de Teposcolula, Oaxaca. Recientemente, se localizó otra población en el límite estatal entre Oaxaca y Puebla por la carretera federal 125.

Para otras especies se han registrado varias poblaciones, sin embargo todas restringidas a un área reducida. Agrupadas en esta condición se encuentran $T$. alpestris ssp. alpestris, T. alpestris Molseed ssp. obtusa Molseed, T. chiapensis Molseed ex Cruden, T. dugesii S. Watson, T. durangensis Molseed, T. flammea, T. galanthoides Molseed, T. huajuapanensis Molseed ex Cruden, T. inusitata, T. martinezii Calderón, T. molseediana, T. pulchella B. L. Rob. y T. seleriana (Loes.) Ravenna (Molseed, 1970; Cruden, 1968, 1975; Espejo-Serna y López-Ferrari, 1996a, 1996b; Rodríguez et al., 1996).

Algunas especies de Tigridia tienen una distribución geográfica amplia y, aunque sus poblaciones son poco frecuentes, el número de individuos que las forman es por lo general elevado. En esta categoría se encuentran $T$. augusta Drapiez, T. ehrenbergii ssp. ehrenbergii, T. hallbergii Molseed ssp. hallbergii, T. immaculata, T. meleagris (Lindl.) G. 
Nicholson, T. mexicana, T. multiflora (Baker) Ravenna, T. vanhouttei ssp. vanhouttei y $T$. orthantha (Molseed, 1970; Espejo-Serna y López-Ferrari, 1996a, 1996b; Rodríguez et al., 1996). Por último, T. pavonia es la especie más ampliamente distribuida. En México, crece a lo largo de la Sierra Madre Occidental, Sierra Madre Oriental, Eje Volcánico Transversal, Sierra Madre del Sur y Sierra Madre de Chiapas (Molseed, 1970).

El objetivo del presente documento es dar a conocer la ubicación de nuevas poblaciones de T. bicolor Molseed, T. matudae Molseed y T. vanhouttei (Baker) Espejo \& López-Ferrari ssp. roldanii Molseed, registradas anteriormente sólo de sus respectivas localidades tipo. Las colectas botánicas se llevaron a cabo durante los veranos de 1996 y 2000 con el fin de ampliar la información acerca de la distribución geográfica de la tribu Tigridieae y obtener semillas, bulbos y hojas para el estudio filogenético del grupo. Los viajes fueron planeados con base en los datos obtenidos de ejemplares de herbario, referencias bibliográficas, sugerencias de otros botánicos y experiencia de colectas anteriores de los propios autores. Se puso especial interés en las localidades tipo registradas por Calderón de Rzedowski, 1987; Cruden, 1968, 1971, 1975; López-Ferrari y Espejo-Serna, 1994; Goldblatt y Howard, 1992; McVaugh, 1989; Molseed, 1968, 1970; Molseed y Cruden, 1968, 1969; Ravenna, 1974; y Rodríguez et al., 1996.

\section{RESULTADOS}

Se localizaron nuevas poblaciones de $T$. bicolor, $T$. matudae y $T$. vanhouttei ssp. roldanii en los estados de Oaxaca, Morelos y Puebla, respectivamente. Los ejemplares de respaldo fueron depositados en los herbarios de la Universidad de Guadalajara (IBUG) y de la Universidad de Wisconsin (WIS). Más duplicados serán distribuidos próximamente.

Tigridia bicolor Molseed, Univ. Calif. Publ. Bot. 54: 94-96, f. 29, 19; lám. 6c. 1970. TIPO: Oaxaca, at km 376 on route 190, ca. $26.5 \mathrm{~km}$ SE of Huajuapan de León, $2270 \mathrm{~m}$, July 21, 1966, E. Molseed 471 (UC, isotipo en GH). septiembre.

Fenología: Florece en los meses de julio y agosto. Fructifica de agosto a

Hábitat: Tigridia bicolor crece en el matorral xerófilo y el bosque de Quercus. Molseed (1970) menciona que T. bicolor es la especie conocida con el hábitat más árido. La población localizada en el municipio de Santiago Tejupan, Oaxaca, crece en matorral xerófilo, en paredones a orillas de la carretera a lo largo de un cañón. En esta localidad son comunes individuos de Ferocactus macrodiscus (Martius) Britton \& Rose, Agave spp., Hechtia spp., Mammillaria spp. y Senecio spp. Existe un estrato arbóreo formado por encinos aislados cubiertos con Tillandsia usneoides (L.) L. La otra población se localiza en el municipio de Teposcolula, Oaxaca, creciendo en el bosque de Quercus y Juniperus. Desafortunadamente, se trata de una zona muy perturbada por el pastoreo de cabras. En consecuencia, las plantas crecen sólo en lugares de muy difícil acceso y bajo la protección de grandes rocas y encinos. 
Distribución: Todas las poblaciones conocidas crecen en la región mixteca, en Oaxaca (Fig. 1).

Ejemplares revisados: Oaxaca: municipio de Santiago Tejupan, $5 \mathrm{~km}$ al NE de Santiago Tejupan sobre la carretera a San Cristóbal Suchixtlahuaca, alt. $2200 \mathrm{~m}, 17^{\circ} 41^{\prime} \mathrm{N}$, 97²3' W, 15.VII.1996, A. Rodríguez et al. 2845 (IBUG, WIS); 27.VIII.2000, A. Rodríguez et al. 2939 (IBUG); municipio de Teposcolula, camino de ascenso hacia la estación de microondas de Yacuden, $62 \mathrm{~km}$ al SE de Huajuapan de León por la carretera federal 190, $1 \mathrm{~km}$ al $\mathrm{E}$ de la intersección con la carretera federal 125 hacia Santiago Pinotepa Nacional, alt. $2200 \mathrm{~m}, 1^{\circ} 33^{\prime} \mathrm{N}, 9^{\circ} 24^{\prime} \mathrm{W}, 15 . \mathrm{VII} .1996$, A. Rodríguez et al. 2843 (IBUG, WIS).

Discusión: Las flores de $T$. bicolor son erectas y extendidas, de 1.5 a $2.5 \mathrm{~cm}$ de diámetro. Los tépalos son conniventes en forma de copa en la base, de color marrón obscuro y la parte distal es amarilla. Las ramas del estilo son filiformes y presentan un mucrón prominente entre ellas (Molseed, 1970). Tigridia bicolor se conocía sólo de la localidad tipo (Molseed, 1970; Espejo-Serna y López-Ferrari, 1996a, 1996b). Molseed (1970) registró su presencia a $26.5 \mathrm{~km}$ al SE de Huajuapan de León por la carretera federal 190, en el punto más alto de esta porción de la Sierra Mixteca, aproximadamente a $1 \mathrm{~km}$ al NE de Santa María Tutla. En los viajes de campo de los autores en 1995 y 1996, se intentó localizar esta población con el fin de recolectar material pero no se logró el objetivo. Actualmente, la zona se utiliza para el pastoreo de cabras y probablemente la especie ha sido extirpada allí. Por el contrario, la población del municipio de Santiago Tejupan es abundante, la mayoría de las plantas se encontraron en floración y algunas con frutos inmaduros. Las flores de T. bicolor tienen un olor fétido. Se observaron moscas y avispas libando sobre los nectarios y es probable que estos insectos sean sus polinizadores. El hábitat muestra disturbio, en apariencia como resultado del pastoreo. Esta especie está sujeta a protección especial de acuerdo con la Norma Oficial Mexicana PROY-NOM-ECOL-059-2000 (Anónimo, 2000).

Tigridia matudae Molseed, Univ. Calif. Publ. Bot. 54: 83-87, f. 24; lám. 5d. 1970. TIPO: México, route 130 SE of Toluca between km 26 and 27, $2980 \mathrm{~m}$, August 27, 1966, E. Molseed 547 (UC, isotipos en GH e ICF).

Fenología: Florece de agosto a septiembre y fructifica en septiembre.

Hábitat: De acuerdo con Molseed (1970), T. matudae crece en lugares sombreados dentro del bosque de pino. En 1996, A. Rodríguez realizó un viaje a la supuesta localidad tipo (ver discusión). En esa ocasión se observaron tres individuos en estado vegetativo y uno más a punto de florecer, que se utilizó para la determinación de la especie. Las plantas se encontraron sólo en paredones a lo largo de la brecha que conduce a Peñuelas. La zona está cubierta por bosque de Abies y Pinus. En el estrato herbáceo son frecuentes individuos de Solanum verrucosum (Schltdl.), S. demissum Lindl., Cirsium sp., Geranium sp., Crotalaria sp., Fuchsia sp., Stevia sp., Penstemon sp. y Castilleja sp. además de muchos musgos. La nueva población, encontrada en el estado de Morelos, 
crece también a lo largo de los paredones de la carretera, en un área cubierta por bosque de Abies con algunos pinos y encinos.

Distribución: Tigridia matudae se conocía únicamente del municipio de Zinacantepec en el estado de México. En este trabajo se registra por primera vez para el estado de Morelos (Fig. 1).

Ejemplares revisados: Estado de México: municipio de Zinacantepec, km 27.5 de la carretera Toluca-Valle de Bravo y $100 \mathrm{~m}$ por la brecha a Peñuelas, alt. $3000 \mathrm{~m}$, 1909'30" N 9951'06" W, 11.VIII.1996, A. Rodríguez 2898 (IBUG); Morelos: municipio de Huitzilac, Sierra de Zempoala, Parque Nacional Lagunas de Zempoala, cerca del vivero forestal, $\mathrm{km} 11$ de la carretera estatal Núm. 4 Huitzilac-Coatepec, bosque de Abies con pinos y encinos, alt. $2900 \mathrm{~m}, 19^{\circ} 02^{\prime} \mathrm{N}, 9^{\circ} 18^{\prime} \mathrm{W}, 28 . \mathrm{VIII} .2000$, A. Rodríguez et al. 2938 (IBUG).

Discusión: Tigridia matudae tiene flores erectas, extendidas, de color azul-violeta pálido con un patrón irregular de manchas. Las ramas del estilo son muy cortas, canaliculadas y presentan un pequeño mucrón en su base (Molseed, 1970). Las referencias de Molseed (1970) respecto a la localidad tipo de T. matudae son imprecisas. De acuerdo con este autor, T. matudae fue colectada entre los kilómetros 26 y 27 de la carretera federal 130 al sureste de Toluca. Sin embargo, la carretera de este número sale de Toluca con dirección noreste hacia Naucalpan. En el kilómetro 26.5 se localiza el puerto El Guarda, lugar cubierto por bosque de Abies y Pinus, muy perturbado debido al sobrepastoreo (Rodríguez, obs. pers., 1995). Por otro lado, la carretera federal 134 sale de Toluca con dirección suroeste hacia Temascaltepec y en el kilómetro 27.5 se encuentra la brecha que conduce al poblado de Peñuelas. Como ya se indicó, el 11 de agosto de 1996 se colectó en la zona y se encontraron cuatro individuos que se determinaron como T. matudae. Otra visita a la misma zona se realizó el 27 de agosto de 2000. En esa ocasión se buscó a lo largo de la brecha a Peñuelas y entre los kilómetros 22 y 27.5 de la carretera 134, sin embargo no se localizó ninguna planta. La nueva localidad de $T$. matudae fue encontrada aproximadamente a $55 \mathrm{~km}$ al sureste de Toluca a lo largo de la carretera estatal 4 entre Coatepec y Huitzilac. La población consta de numerosos individuos restringidos exclusivamente a los paredones de la carretera.

Tigridia vanhouttei (Baker) Espejo \& López-Ferrari ssp. roldanii Molseed, Univ. Calif. Publ. Bot. 54: 100-101. 1970. TIPO: Hidalgo, Tecocotla, 1 mile S Ciudad Sahagún, in a wheat field on a knoll among Agave and Opuntia, ca. 2400 m, August 14, 1962, Molseed, Roldan \& Keator 122 (UC).

Fenología: Florece en agosto y fructifica en septiembre.

Hábitat: La población, formada por muchos individuos, se ubica en paredones a lo largo de la carretera, junto a cultivos de maíz, rodeados por un bosque de pino y encino. 


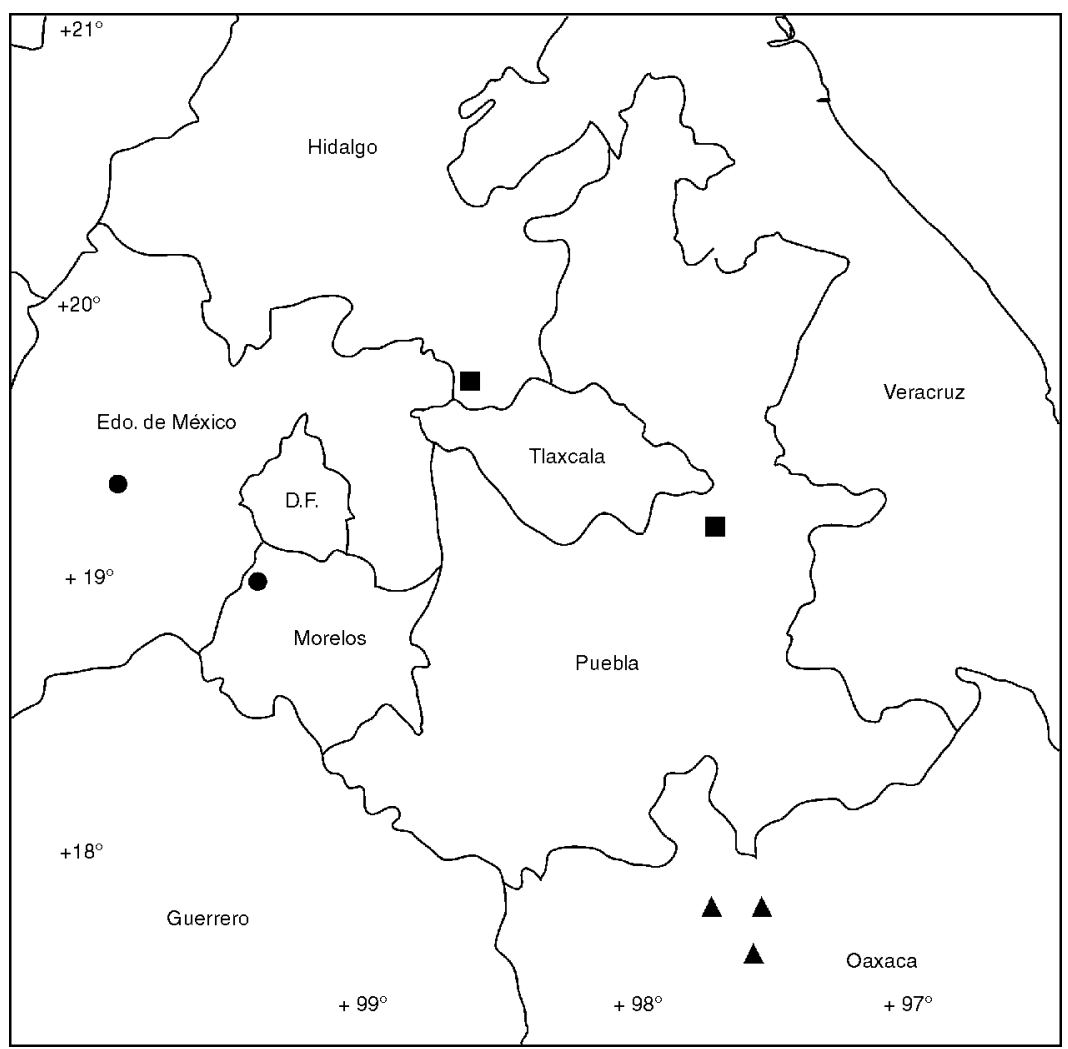

Fig. 1. Localidades conocidas de Tigridia bicolor ( $\mathbf{\Delta})$, T. matudae (-) y T. vanhouttei ssp. roldanii (ם).

Distribución: Tigridia vanhouttei ssp. roldanii se conocía sólo del estado de Hidalgo. En este artículo se registra por primera vez para Puebla (Fig. 1).

Ejemplares revisados: Hidalgo: municipio de Tepeapulco, $1 \mathrm{~km}$ E de Tepeapulco por la carr. Tepeapulco-Apan, $2450 \mathrm{~m}, 1^{\circ} 46^{\prime} \mathrm{N}, 98^{\circ} 32^{\prime} \mathrm{W}, 30 . \mathrm{VII} .1996$, A. Rodríguez y C. Martinelli 2860 (IBUG, WIS); Hidalgo: municipio. de Cd. Sahagún, cerro de Santa Lucía, al E de Cd. Sahagún, $2500 \mathrm{~m}, 19^{\circ} 46^{\prime} \mathrm{N}, 98^{\circ} 33^{\prime} \mathrm{W}, 30 . \mathrm{VII} .1996$, A. Rodríguez y C. Martinelli 2863 (IBUG, WIS); Puebla: municipio de Mazapiltepec, carretera federal 140 entre Acatzingo y San Salvador el Seco, $500 \mathrm{~m}$ al SW del cruce con la vía del ferrocarril México-Córdoba y la carretera a Soltepec, $2300 \mathrm{~m}, 19^{\circ} 04^{\prime} \mathrm{N}, 97^{\circ} 39^{\prime} \mathrm{W}, 7 . \mathrm{VIII} .1996, A$. Rodríguez 2882 (IBUG, WIS). 
Discusión: Típicamente, este taxon posee flores péndulas (Molseed, 1970), tépalos interiores ovalados y un mucrón inserto por abajo de la unión de las ramas del estilo. En la población localizada en el estado de Puebla, la mayoría de las flores eran péndulas pero se observaron algunas extendidas y hasta erectas. Sin embargo, su identificación y afiliación con la ssp. roldanii es clara al analizar la forma de los tépalos interiores y el mucrón. Otra planta morfológicamente similar a $T$. vanhouttei ssp. roldanii es $T$. ehrenbergii ssp. ehrenbergii. Ambos taxones presentan flores péndulas pero difieren en el tamaño relativo de los tépalos interiores. En $T$. vanhouttei ssp. roldanii la longitud de los tépalos interiores es de tres cuartas partes de la longitud de los tépalos exteriores. En contraste, los tépalos interiores de $T$. ehrenbergii ssp. ehrenbergii apenas alcanzan la mitad del largo de los tépalos exteriores. Además, las flores de T. ehrenbergii ssp. ehrenbergii son fétidas, mientras que las de $T$. vanhouttei ssp. roldanii son inodoras.

\section{CONCLUSIONES}

El conocimiento sobre el número de especies de Tigridia en México y su distribución es incompleto. Es muy probable que algunas consideradas como raras 0 conocidas de una sola localidad, tengan una distribución más amplia. Prueba de lo anterior es el informe de las poblaciones recientemente encontradas de $T$. matudae, $T$. bicolor y $T$. vanhouttei ssp. roldanii que aquí se presenta. Son necesarias exploraciones botánicas más intensas para determinar con mayor precisión el número de taxa existentes y los ámbitos de distribución de las mismas. En general, muchos representantes de Tigridia se ven favorecidos por la construcción de carreteras y caminos, las plantas a menudo se desarrollan en los paredones que con frecuencia bordean estas vías. Sin embargo, es evidente la vulnerabilidad de algunas especies ante otras actividades humanas, en particular el pastoreo y el crecimiento de las zonas urbanas.

\section{AGRADECIMIENTOS}

La exploración efectuada en 1996 se realizó con la ayuda económica de la Universidad de Guadalajara y la Fundación Davis, Departamento de Botánica, Universidad de Wisconsin. El primer autor agradece a Ofelia Vargas, Eduardo Villegas, Chris Martinelli y Raymundo Ramírez Delgadillo su ayuda durante la colecta del mismo año.

\section{LITERATURA CITADA}

Anónimo. 2000. Diario Oficial de la Federación. Norma Oficial Mexicana PROY-NOM-059-ECOL2000. $55 \mathrm{pp}$.

Calderón de Rzedowski, G. 1987. Tigridia martinezii, una especie nueva de iridáceas del estado de Hidalgo (México). Bol. Soc. Bot. México 47: 3-6.

Cruden, W. R. 1968. Three new species of Tigridia (Iridiaceae) from Mexico. Brittonia 20: 314-320.

Cruden, W. R. 1971. The systematics of Rigidella (Iridaceae). Brittonia 23: 217-225.

Cruden, W. R. 1975. New Tigridieae (Iridaceae) from Mexico. Brittonia 27: 103-109. 
Acta Botanica Mexicana (2003), 62: 1-8

Espejo-Serna, A. y A. R. López-Ferrari. 1996a. Las monocotiledóneas mexicanas, una sinopsis florística. 1. Lista de referencia. Parte VI. Consejo Nacional de la Flora de México, A. C., Universidad Autónoma Metropolitana Iztapalapa, Comisión Nacional para el Conocimiento y Uso de la Biodiversidad. México, D.F. 116 pp.

Espejo-Serna, A. y A. R. López-Ferrari. 1996b. Comentarios florístico-ecológicos sobre las iridáceas mexicanas. Acta Bot. Mex. 34: 25-47.

Goldblatt, P. y T. M. Howard. 1992. Notes on Alophia (Iridaceae) and a new species, A. veracruzana, from Veracruz, Mexico. Ann. Missouri Bot. Gard. 79: 901-905.

Goldblatt, P., J. C. Manning y P. Rudall. 1998. Iridaceae. In: Kubitzki, K. (ed.). The families and genera of vascular plants III. Springer. Berlín. pp. 295-333.

Henrich, J. E. y P. Goldblatt. 1994. Iridaceae. In: Davidse, G., M. Sousa. y A. Q. Charter (eds.). Flora Mesoamericana. Volumen 6. Instituto de Biología, Universidad Nacional Autónoma de México, México, D.F. pp. 71-80.

López-Ferrari, A. R. y A. Espejo-Serna. 1994. Tigridia estelae (Iridaceae; Tigridieae), a new species from Durango, Mexico. Novon 4: 386-390.

McVaugh, R. 1989. Bromeliaceae to Dioscoreaceae. In: Anderson, W. R. (ed.). Flora Novo-Galiciana. Volumen 15. The University of Michigan Herbarium. Ann Arbor, Michigan. pp. 294-347.

Molseed, E. 1968. Fosteria, a new genus of Mexican Iridaceae. Brittonia 20: 232-234.

Molseed, E. 1970. The genus Tigridia (Iridaceae) of Mexico and Central America. Univ. Calif. Publ. Bot. 54: 1-113.

Molseed, E. y R.W. Cruden. 1968. A new species of Nemastylis (Iridaceae) from Mexico. Brittonia 20: 235-237.

Molseed, E. y R.W. Cruden. 1969. Sessilanthera, a new genus of American Iridaceae. Brittonia 21: 191-193.

Ravenna, P. F. 1974. Cobana, a new genus of Central American Iridaceae. Bot. Not. 127: 104-108.

Rodríguez, A. 1999. Molecular and morphological systematics of the "tiger-flower" group (Tribe Tigridieae: Iridaceae), biogeography and evidence for the adaptive radiation of the subtribe Tigridiinae. Tesis Doctoral, Univeristy of Wisconsin. Madison, Wis. 225 pp.

Rodríguez, A., O. Vargas, E. Villegas y K. J. Sytsma. 1996. Nuevos informes de iridáceas (Tigridieae) en Jalisco. Bol. Inform. Inst. Bot. Univ. Guadalajara 4: 39-47. 